\title{
DILEPTON AS AN ELECTROMAGNETIC PROBE FOR QGP IN HEAVY ION COLLISION
}

\author{
P. K. SETHY \\ Department of Physics, University of Delhi, Delhi, India
}

\begin{abstract}
We discuss the dilepton production mechanism in heavy ion collision considering the effect of chemical potential dependent quark mass based on quasi-particle model. We also calculate the production rate of dilepton using the thermal quark mass for different chemical potentials.
\end{abstract}

KEYWORDS: Dilepton, quark mass, quasi particle

Received: Oct 03, 2017; Accepted: Oct 23, 2017; Published: Oct 29, 2017; Paper Id.: IJPROCT20173

\section{INTRODUCTION}

In the past few years, a lot of work has been done on electromagnetic response of quark gluon plasma (QGP) in heavy ion collision. Quantum chromo dynamics (QCD) has been unarguably the most suitable theory to understand the strong interaction. QCD too augment the experimental heavy ion collision and various properties of dense astrophysical and cosmological matter. QCD- the theory predicts the possible phase transition from a confined state to a deconfined state in the early universe or to be specific just few zepto seconds after the big bang [1]. The exotic state of matter produced after the phase transition is referred to as QGP and it is believed that in this plasma the quarks and gluons are asymptotically free. The state-of-the-art experimental facilities at Brookhaven National Laboratory (BNL) and European Council for Nuclear research (CERN) have been very successful in achieving very high energy, density and temperature which paved the way in confirming the formation of QGP. Although the theory predicts and indicates the possible formation of such an exotic state of matter under such special circumstances, the detection of QGP has been a challenging task for the experimentalists around the globe even if it is believed and widely accepted that QGP is formed in heavy ion collision [2-4]. However, there are several favourable signals like photon emission, strangeness enhancement [5], $J / \psi$ suppression [6], electromagnetic emission like dilepton production and photon production [7-10].

The electromagnetic emission is useful because they carry information without further interaction from the core of the plasma to the detector. The dileptons and photons have been widely believed and accepted as prominent signatures of the QGP. Two heavy ions i.e. their nuclei approach towards each other and collide with huge energy. The collision completely dismantles the nuclei and eventually the substructure is exposed in pre-equilibrium stage. Further, in the due process of thermalization, the plasma goes through several stages and eventually freeze-out takes place. In chemical freeze-out the inelastic scatterings cease whereas in kinetic freeze-out the elastic scatterings cease. 


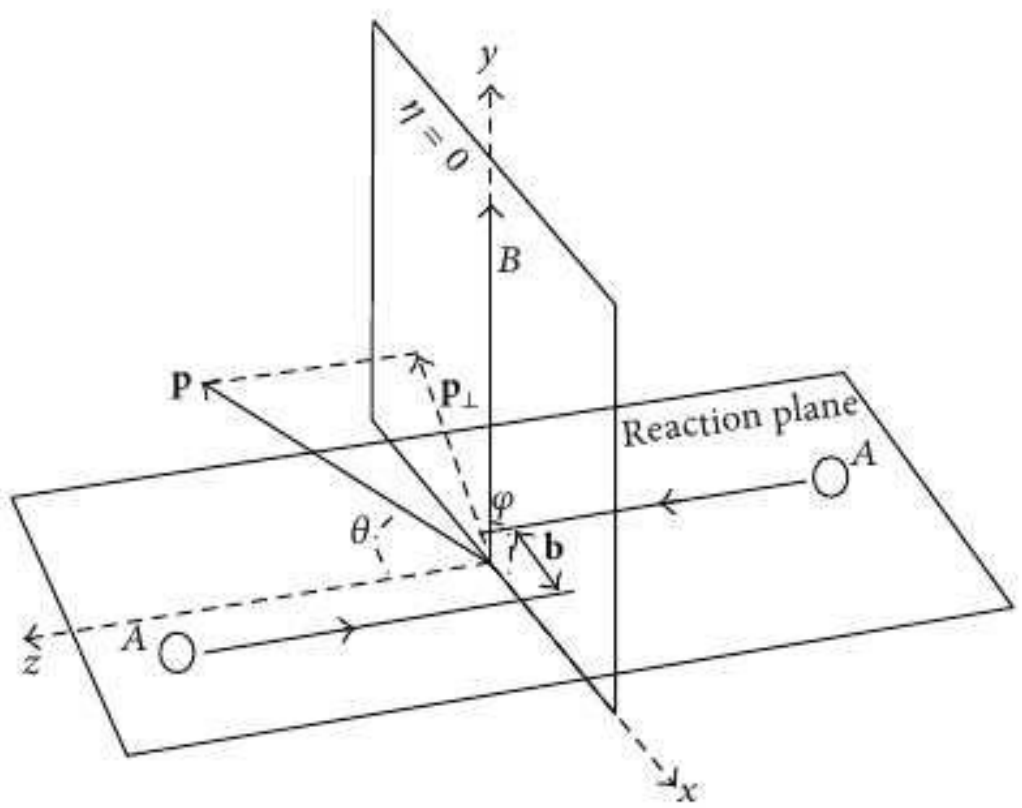

Figure 1: Schematic diagram of collision plane

\section{Dileptons production from a QGP in heavy-ion collision}

Dileptons are produced from several sources. But here we will discuss the interaction of quarks (or antiquarks) through the mediation of a virtual photon results in the production of a lepton pair. Here we consider the processes like $q \bar{q} \rightarrow l^{+} l^{-}$or $\overline{q(q)} g \rightarrow q(\bar{q})+l^{+} l^{-}$as the most prominent one. Nevertheless in recent times, experts [15] have investigated Compton like processes such as $q(\bar{q}) g \rightarrow q(\bar{q}) l^{+} l^{-}, g g \rightarrow q(\bar{q}) l^{+} l^{-}$in the heavy-ion collision. Here we exclusively consider $q \bar{q} \rightarrow l^{+} l^{-}$process because of the larger possibility of lepton pair in the intermediate mass region. There are other sources of dilepton in heavy ion collision. Depending on the invariant mass, there can be broadly three regions of invariant mass such as low mass region (LMR) in which the invariant mass is less than phi $(\varphi)$ meson. Intermediate mass region (IMR) includes the invariant mass between the $\varphi$ meson and $J / \psi$ mass and it is considered to be the true source of dilepton. High mass region (HMR) includes invariant mass above $3.8 \mathrm{GeV}$ which involves Drell-Yan and B-meson decays. It is also reported that the effect of electromagnetic reaction receives $O\left(\alpha^{2} \alpha_{s}\right)$ correction from strong interaction process. In the calculation we also consider the chemical potential dependent on the distribution function and quark mass but excluded the dependency of chemical potential on cross section even though many conjectures of chemical potential in the cross section are found in various literatures. QGP is described by the system of massive non-interacting quasi-particles where the mass of these quasi-particles is temperature dependent. The interaction of quarks and gluons with the surrounding matter helps the quasi-particles to gain mass. The modified quasi-particle mass is given by [11, 17]

$$
m_{\text {eff }}^{2}=m_{c}^{2}+\sqrt{2} m_{c} m_{q}+m_{q}^{2}
$$

where $m_{c}$ is the current mass of the quark and $m_{q}$ is the thermal mass of the quark. 
The dilepton production rate has been obtained primarily in the relevant range of mass region based on the reports of low mass dileptons. Although the process like Compton-scattering, annihilation with scattering are useful sources for production rate, we considered quark-antiquark annihilation process. This consideration is due to the IMR region of the thermal quark-antiquark annihilation as a measurable signal in the intermediate mass region (IMR). Here, we explicitly exclude the dilepton contribution from the hadronic phase in low invariant mass regime. The objective of this paper is to calculate dilepton production rate in a QGP with a modified quark mass. With the above approximations, the dilepton production rate can be written as,

$$
\frac{d N}{d M^{2} d^{4} x}=\frac{5 \alpha^{2}}{18 \pi^{3}} T M \lambda_{q}^{2} \times\left(1+\frac{\left.2\left(m_{c}^{2}+\sqrt{2} m_{c} m_{q}\right)+m_{q}^{2}\right)}{M^{2}}\right) K_{1}(M / T)
$$

The quark mass as a function of chemical potential [11]

$$
m_{q}^{2}\left(T, \mu_{q}\right)=\gamma_{q} \frac{16 \pi}{\left(33-2 N_{f}\right)} \frac{1}{\ln \left(1+\frac{k^{2}}{\Lambda^{2}}\right)}\left(T^{2}+\frac{\mu_{q}^{2}}{\pi^{2}}\right)
$$

which is called as thermal quark mass. $g^{2}=4 \pi \alpha_{s}$ and $\alpha_{s}$ is the QCD coupling factor where,

$$
\alpha_{s}=\frac{4}{\left(33-2 N_{f}\right) \ln \left(1+\frac{k^{2}}{\Lambda^{2}}\right)}
$$

$\alpha_{\mathrm{s}}=0: 15 \mathrm{GeV} . \Lambda$ is QCD parameter and $N_{f}$ is the quark flavour and $k$ is the momentum value. Using an expression given in Ref. [12-13] the dilepton production rate expressed as,

$$
\frac{d N}{d M^{2} d y}=\frac{5 \alpha^{2} R^{2}}{18 \pi^{2}} \times \int\left(1+\frac{2 m_{e f f}^{2}}{M^{2}}\right) \lambda_{q}^{2}(\tau) G(z, \tau) T(\tau) \tau d \tau
$$

\section{RESULTS}

The dilepton yield is calculated using the modified effective quark mass. The results of dilepton emission through quasiparticle model are shown in which the quark mass is a function of temperature and chemical potential. The results are displayed in the figure (2). The dilepton production rates are obtained for the relevant range of the invariant mass. Fig. (2) illustrates the production rate of dilepton for different values of temperature and chemical potential at different initial temperatures ranging from $T_{0}=0: 25 \mathrm{GeV}$ to $T_{0}=0: 57 \mathrm{GeV}$. The dilepton yield is obtained for the critical temperature $\mathrm{T}_{\mathrm{c}}$ of $0.17 \mathrm{GeV}$ and the production rate is found to be increasing with the increase in temperature $T_{0}$ and chemical potential. 


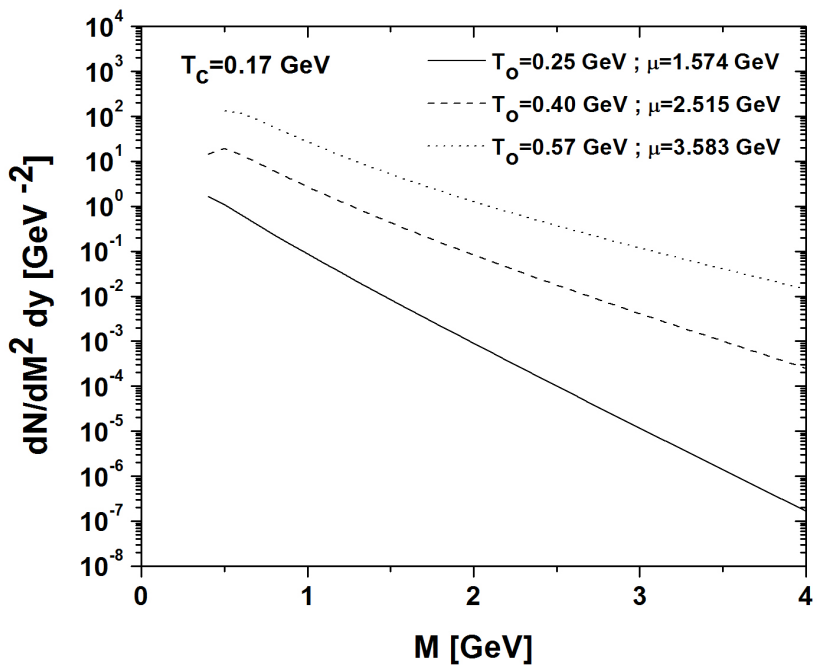

Figure 2: Dilepton production rate $\mathrm{dN} / \mathrm{dM}^{2} \mathrm{dy}$ is plotted for different chemical potentials.

\section{REFERENCES}

1. J. W. Harris and B M M uller, The search for the quark-gluon plasma, Ann. Rev. Nucl and Par. Sc., 46 (1996) 71.

2. S. Nagamiya, Experimental overview, Nucl. Phys. A 544, (1992) 5c.

3. W. Busza, Fragmentation Data in Hadron - Nucleus Collisions: Implications about Nuclear Stopping Power, Nucl. Phys. A 418, (1984) 635c.

4. O. Hansen, Proc. of the 20th Int. Workshop on Gross Production of Nuclei and Nuclear Excitations (Hirschegg, Austria) H. Ferdmeier (1992).

5. P. Muthu Kumar \& S. Jerome, "Effect of Eccentricity Pin Geometry on the Fabrication of Surface Composite (Al6061-T6 /Sic) by Friction Stir Processing “, International Journal of Mechanical and Production Engineering Research and Development (IJMPERD), Vol. 8, Issue 6, pp. 249-254

6. Benjamin Tawiah \& Benjamin K. Asinyo, “Advances in Spun-Dyeing of Regenerated Cellulose Fibers “, BEST: International Journal of Management, Information Technology and Engineering (BEST: IJMITE), Vol. 4, Issue 2, pp. 65-80

7. Sumit Kumar Gupta, "Study of Behaviour and Nature of Doping Holes in Under Doped and Overdoped Regimes Cuprate Perovskites "International Journal of Applied and Natural Sciences (IJANS), Vol. 3, Issue 3, May 2014, 15-36

8. J. Rafelski, B. Muller, Strangeness production in the quark-gluon plasma, Phys. Rev. Lett., 48 (1982) 1066.

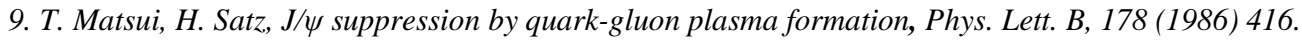

10. E. Shuryak, Quantum chromodynamics and the theory of superdense matter, Phys. Rep. 61, (1980) 71.

11. K. Kajantie, J. Kapusta, L. McLerran, A. Mekjian, Dilepton emission and the QCD phase transition in ultrarelativistic nuclear collisions, Phys. Rev. D, 34 (1986) 2746.

12. S. Y. Wang and D. Boyanovsky, Enhanced photon production from quark-gluon plasma: Finite-lifetime effect, Phys. Rev. D, 63 (2001) 051702.

13. Y. Kumar, P. Jain, Phenomenological modeling of the photon production rate from QGP at finite chemical potential, Int. J. Mod. Phys. A, 30 no. 33(2015)1550196. 
14. Y. Kumar, EPJ Web of Conf. 182, 02070 (2018).

15. A. Bialas, W. Czyz_, Boost-invariant Boltzmann-Vlasov equations for relativistic quark-antiquark plasma, Phys. Rev. D, 30 (1984) 2371.

16. O. Hansen, Proc. of the 20th Int. Workshop on Gross Production of Nuclei and Nuclear Excitations (Hirschegg, Austria) Ed H. Ferdmeier (1992)

17. Inácio Malmonge Martin, Marcelo Pego Gomes \& Anatoli A. Gusev, "Low Energy Gamma Rays Measurements During January to February 2017 in São José Dos Campos, Sp, Brazil Region “, IMPACT: International Journal of Research in Engineering \& Technology (IMPACT: IJRET), Vol. 5, Issue 3, pp. 21-26

18. D. Dutta et al, D Dutta, K Kumar, A K Mohanty, $R$ K Choudhury Chemical equilibration and thermal dilepton production from the quark gluon plasma at finite baryon density, Phys. Rev. C 60 (1999) 014905.

19. Z He, J Long, W Jiang, Y Ma, B Liu, Intermediate mass dileptons from a chemically non-equilibrated quark- gluon plasma, Nucl. Phys. A 724 (2003) 477.

20. J. L. Long, Z. J. He, Y. G. Ma and B. Liu, Hard photon production from a chemically equilibrating quark-gluon plasma with finite baryon density at one loop and two loop, Phys. Rev.C, 72 (2005) 064907.

21. P. K. Srivastava, S. K. Tiwari, and C. P. Singh, QCD critical point in a quasiparticle model, Phys. Rev. D 82 (2010) 014023. 

